\title{
Seminare / Séminaires / Seminari 2013
}

\section{Praxiseröffnung/-übernahme}

Das Seminar richtet sich an Ärztinnen und Ärzte, welche vor einer Praxiseröffnung (Einzel-/Gruppenpraxis), dem Einstieg in eine Gruppenpraxis oder vor einer Praxisübernahme stehen.

\section{Themen}

- Juristische Aspekte (Praxisbewilligung, Zulassung zur Sozialversicherung, Vertragswesen)

- Gesellschaftsformen/Ehe- und Erbrecht (Privat-/Geschäftsvermögen, Güterstand, Erbschaftsplanung)

- Praxiseinrichtung (Inneneinrichtung, Kostenberechnung)

- Praxisadministration (Leistungserfassungsund Abrechnungssysteme)

- Bewertung einer Arztpraxis (Berechnung Inventarwert und Goodwill als Verhandlungsbasis)

- Finanzierung der Arztpraxis (Businessplan, Kredite, Absicherungsmöglichkeiten)

- Versicherungen/Vorsorge/Vermögen (Personen- und Sachversicherungen, Vorsorgeplanung)

\section{Sponsoren}

Die Kosten werden durch diverse Sponsoren (siehe www.fmhservices.ch) gedeckt.

\begin{tabular}{|lll}
$\begin{array}{l}\text { Daten } \\
\text { K04 }\end{array}$ & $\begin{array}{l}\text { Donnerstag, } \\
\text { 5. September 2013 } \\
\text { 9.00-16.30 Uhr }\end{array}$ & $\begin{array}{l}\text { Zürich } \\
\text { Volkshaus }\end{array}$ \\
\hline K05 & $\begin{array}{l}\text { Donnerstag, } \\
\text { 7. November 2013 } \\
\text { 9.00-16.30 Uhr }\end{array}$ & $\begin{array}{l}\text { Hotel Victoria } \\
\text { Basel }\end{array}$ \\
\hline
\end{tabular}

\section{Praxisübergabe}

Das Seminar richtet sich an zukünftige Praxisübergeber/innen. Idealtermin: 5-10 Jahre vor geplanter Übergabe (aus steuer- und vorsorgeplanerischen Gründen).

\section{Themen}

- Juristische Aspekte (Praxisübergabevertrag, allg. Vertragswesen, Übergabe der Krankengeschichten)

- Nachfolgeplanung und Bewertung einer Arztpraxis (projektorientiertes Vorgehen in der Nachfolgeplanung, Berechnung Inventarwert und Goodwill als Verhandlungsbasis)

- Versicherungen/Vorsorge/Vermögen (Übergabe/Auflösung von Versicherungsverträgen, Pensions- und Finanzplanung)

- Steuern (Steueraspekte bei der Praxisübergabe: Optimierung der steuerlichen Auswirkungen, Liquidations- und Grundstückgewinnsteuer, Bestimmung des optimalen Übergabezeitpunktes)

\section{Sponsoren}

Die Kosten werden durch diverse Sponsoren (siehe www.fmhservices.ch) gedeckt.

\begin{tabular}{|lll}
$\begin{array}{l}\text { Daten } \\
\text { K09 }\end{array}$ & $\begin{array}{l}\text { Donnerstag, } \\
\text { 12. September 2013 }\end{array}$ & $\begin{array}{l}\text { Zürich } \\
\text { Volkshaus }\end{array}$ \\
\hline K10 & $\begin{array}{l}\text { Donnerstag, } \\
\text { 14. November 2013 }\end{array}$ & $\begin{array}{l}\text { Basel } \\
\text { Hotel Victoria }\end{array}$ \\
& 13.30-18.00 Uhr & \\
\hline
\end{tabular}

Finanz- und Steuerplanung

Das Seminar richtet sich an Praxiseröffner/innen, Praxisübernehmer/innen sowie an bereits praxistätige Ärztinnen und Ärzte.

\section{Themen}

- Finanzplanung (Businessplan, buchhalterische Massnahmen vor Praxiseröffnung/ -übernahme, Standardkontenplan, doppelte Buchhaltung, EDV-unterstützte Buchführungslösung)

- Steuern (Steueraspekte bei Eintritt in die Selbständigkeit, Steuerfallen und Steuerrisiken, optimierte Steuerplanung)

\section{Kosten}

Für FMH Services-Mitglieder kostenlos.

$$
\begin{array}{lll}
\text { Datum } & & \\
\text { K12 } & \begin{array}{l}
\text { Donnerstag, } \\
\text { 19. September } 2013
\end{array} & \text { Bern } \\
& \text { Schmiedstube } \\
& \text { 13.30-18.00 Uhr } &
\end{array}
$$

\section{Praxiscomputerworkshop}

Der Workshop richtet sich an praxiseröffnende sowie an bereits praxistätige Ärztinnen und Ärzte.

\section{Inhalt}

- Anforderungen an ein Praxisinformationssystem (Einführung)

- Evaluationsprozess (projektorientiertes Vorgehen in der Evaluation eines Praxisinformationssystems)

- Präsentation von sechs führenden Praxisinformationssystemen (Leistungserfassung, elektronisches Abrechnen unter Einbezug der TrustCenter, Agendaführung, Statistiken, Laborgeräteeinbindung, elektronische Krankengeschichte, Finanzbuchhaltungslösungen usw.)

\section{Kosten}

Für FMH Services-Mitglieder kostenlos.

\section{Datum}

$\begin{array}{lll}\text { K15 Donnerstag, } & \text { Olten } \\ & \text { 28. November } 2013 & \text { Stadttheater }\end{array}$

\section{Röntgen in der Arztpraxis}

Das Seminar richtet sich an Ärztinnen und Ärzte mit bestehender Praxis und an solche, die vor einer Praxiseröffnung oder Praxisübernahme stehen. Die Teilnehmerzahl ist begrenzt.

\section{Themen}

- Vom konventionellen zum digitalen Röntgen

- Rentabilität Röntgen in der Arztpraxis

- Neue Vorschriften

- Evaluation und Beschaffung neuer oder gebrauchter Anlagen

- Komplette Marktübersicht mit Preisen und Leistungskomponenten

- Praktische Übung (Erstellen und Bearbeiten digitaler Röntgenbilder)

\section{Kosten}

300 CHF (inkl. sämtlicher Kursunterlagen und Verpflegung)

\section{Datum \\ K16 Donnerstag 22. August 2013 Niederscherl 09.30-16.00 Uhr WIROMA AG}

\section{Tarifwerk TARMED - Einführungskurs}

Das Seminar richtet sich an Ärztinnen und Ärzte mit bestehender Praxis und an solche, die kurz vor einer Praxiseröffnung oder Praxisübernahme stehen. Der Einführungskurs vermittelt den Kursteilnehmern die Grundlagen des Tarifwerkes TARMED. Die Teilnehmerzahl ist begrenzt.

Themen

- Fakten (gesetzliche und vertragliche Grundlagen)

- Struktur (Tarifbrowser, Grundstruktur, Regelhierarchie, Leistungsblöcke, Leistungsgruppen)

- Generelle Interpretationen («Allgemeine Grundleistungen», «Hauptleistungen, Zuschlagsleistungen», «Nichtärztliche Leistungserbringung» usw.)

- Parameter einer Tarifposition («Quantitative und Qualitative Dignität», «Ärztliche Leistung AL», «Assistenz», «Raumbelegung» usw.)

- Tarifpositionen aus dem Kapitel 00 Grundleistungen

- Praxislabor und Präsenzdiagnostik (neue Analyseliste)

- Organisationen und Informationsquellen

\section{Kosten}

200 CHF (inkl. Kursunterlagen)

$\begin{array}{lll}\text { Datum } & \\ \text { K62 } & \text { Dienstag, } & \text { Olten } \\ & \text { 17. September } 2013 & \text { Hotel Arte } \\ & \text { 14.00-17.15 Uhr } & \end{array}$




\section{Praxismarketing für Ärzte}

Das Seminar richtet sich an Ärztinnen und Ärzte, welche vor einer Praxiseröffnung stehen oder bereits eine Praxis führen.

\section{Themen}

- Service und Kundenorientierung: Aus einer Arztpraxis wird DIE Praxis.

- Menschen überraschen und hohes Niveau halten

- Telefon, Empfang, Teamentwicklung Werbemöglichkeiten

\section{Kosten}

300 CHF (inkl. sämtliche Kursunterlagen und Verpflegungen)

\section{Datum \\ K64 Mittwoch, Bern \\ 11. September $2013 \quad$ Schmiedstube 13.30-18.00 Uhr}

Telefonseminar für MPA (bzw. Praxisteam) Das Seminar richtet sich an MPAs mit telefonischem Kundenkontakt sowie Auszubildende, die zum professionellen Telefonieren angeleitet werden sollen.

\section{Themen}

- Die medizinische Praxisassistentin als Visitenkarte der Praxis

- Image der Arztpraxis. MPAs repräsentieren die Unternehmenskultur, organisieren die Praxis und sind somit ein wesentlicher Bestandteil für den Unternehmenserfolg.

- Bedeutung des ersten Telefonkontakts

- Richtig telefonieren - eine Anleitung

\section{Kosten}

300 CHF (inkl. sämtliche Kursunterlagen und Verpflegungen)

Praxisteams erhalten einen Rabatt von 20\% pro Teilnehmer/in

\section{Datum \\ K66 \\ $\begin{array}{ll}\text { Mittwoch, } & \text { Bern } \\ \text { 06. November 2013 } & \text { Schmiedstube }\end{array}$ $09.00-16.30 \mathrm{Uh}$}

\section{Crashkurs Versicherungsmedizin}

Das Seminar richtet sich an Ärztinnen und Ärzte, vom Assistenzarzt bis zum Klinikdirektor, vom Grundversorger bis zum Supersuperspezialisten. Das Seminar wird in Zusammenarbeit mit der Schweiz. Gesellschaft der Vertrauens- und Versicherungsärzte (SGV) durchgeführt.

\section{Themen}

- Spielregeln der Versicherungsmedizin von KVG, UVG und IVG anhand von Fallbeispielen

- Gesetze und Verordnungen

- Zuständige Ämter

\section{Kosten}

100 CHF (inkl. sämtliche Kursunterlagen und Verpflegungen)

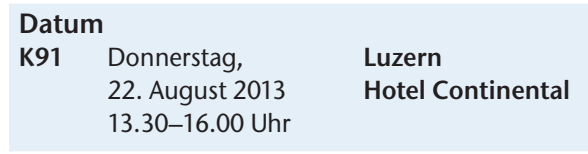

Ouverture et reprise d'un cabinet médical Le séminaire est destiné aux médecins sur le point d'ouvrir un cabinet médical (individuel ou de groupe), de joindre un cabinet de groupe ou de reprendre un cabinet existant.

\section{Contenu}

- Business plan (préparation du plan de financement et crédit d'exploitation, financement par la banque)

- Aménagement (implantation, projet et concept d'aménagement, choix du mobilier budget)

- Estimation d'un cabinet (inventaire et goodwill)

- Administration d'un cabinet médical (dans le cabinet, par la banque)

- Assurances (toutes les assurances à l'intérieur et autour du cabinet)

- Passage du statut de salarié à celui d'indépendant

- Fiscalité

\section{Sponsors}

Les coûts sont pris en charge par divers sponsors (voir www.fmhservices.ch).

\begin{tabular}{|c|c|c|}
\hline \multicolumn{3}{|c|}{ Dates } \\
\hline K22 & $\begin{array}{l}\text { Jeudi, } \\
12 \text { septembre } 2013 \\
17.00-21.30 \mathrm{~h}\end{array}$ & $\begin{array}{l}\text { Lausanne } \\
\text { World Trade Center }\end{array}$ \\
\hline K23 & $\begin{array}{l}\text { Jeudi, } \\
21 \text { novembre } 2013 \\
17.00-21.30 \mathrm{~h}\end{array}$ & $\begin{array}{l}\text { Genève } \\
\text { Crowne Plaza }\end{array}$ \\
\hline
\end{tabular}

\section{Remise d'un cabinet médical}

Le séminaire s'adresse aux médecins désirant remettre un cabinet médical. Idéalement 5-10 ans avant la remise prévue (pour des questions de taxation et prévoyance).

\section{Contenu}

- Aspects juridiques (autour du contrat de remise/reprise)

- Estimation d'un cabinet (inventaire et goodwill)

- Assurances (prévoyance, assurances à l'intérieur et autour du cabinet)

- Conséquences fiscales d'une remise

\section{Sponsors}

Les coûts sont pris en charge par divers sponsors (voir www.fmhservices.ch)

$\begin{array}{lll}\begin{array}{l}\text { Date } \\ \text { K25 }\end{array} & \text { Jeudi, } \\ & \begin{array}{l}\text { Genève } \\ 14.00-21.30 \mathrm{~h}\end{array} & \begin{array}{l}\text { Crowne Plaza } \\ \text { Cr. } 2013\end{array}\end{array}$

Apertura e rilevamento di uno studio medico Il seminario è destinato ai medici in procinto di aprire o di rilevare uno studio medico.

\section{Contenuto}

- Business Plan (preparazione del piano di finanziamento e del credito d'esercizio, prestito bancario)

- Pianificazione (insediamento, progetto e pianificazione, scelta del mobilio, budget)

- Valutazione di uno studio medico (inventario e goodwill)

- Amministrazione di uno studio medico (interna allo studio, rapporti con la banca)

- Assicurazioni (tutte le assicurazioni necessarie interne ed esterne allo studio)

- Passaggio dallo stato di dipendente a quello di indipendente

- Fiscalità

\section{Sponsor}

Diversi sponsor si fanno carico delle spese (si rimanda al sito www.fmhservices.ch).

\section{Data K51}

$\begin{array}{ll}\text { Giovedì, } & \text { Chiasso } \\ 24 \text { ottobre } 2013 & \text { FMH Fiduciaria } \\ \text { dalle } 14.00 \text { alle } 18.00 & \text { Services }\end{array}$

\section{Anmeldung und Auskunft /} Inscription et information / Iscrizioni e informazioni www.fmhservices.ch oder FMH Consulting Services, Cornelia Fuchs, Burghöhe 1, 6208 Oberkirch, Tel. 04192500 77, Fax 0419210586

\section{Hinweis / Remarque / Osservazioni}

Bei sämtlichen Seminaren, bei denen die Kosten teilweise oder gänzlich von Seminarsponsoren gedeckt werden, werden die Teilnehmeradressen den jeweiligen Sponsoren zur Verfügung gestellt. Les adresses des participants aux séminaires dont les coûts sont couverts en partie ou totalement par des sponsors sont communiquées aux sponsors concernés.

Gli indirizzi dei partecipanti ai seminari, i cui costi sono coperti in parte o completamente da degli sponsor, vengono comunicati agli sponsor interessati.

\section{Annullierungsbedingungen / Conditions} d'annulation / Condizioni d'annullamento Bei Abmeldungen oder Fernbleiben werden folgende Unkostenbeiträge erhoben:

Un montant est perçu pour une absence ou une annulation. Il est de:

Un importo verrà rimborsato in caso di assenza o annullamento. Esso sarà di:

- 50 CHF pro Person ab 14 Tage vor Seminarbeginn / par personne dans les 15 jours avant le début du séminaire/ per persona entro i 15 giorni prima dell'inizio del seminario;

- 100 CHF pro Person ab 7 Tage vor Seminarbeginn oder Fernbleiben / par personne dans les 7 jours avant le début du séminaire / per persona entro i 7 giorni prima dell'inizio del seminario. 